\title{
Study of the airborne behavior of cedar pollen and its allergen released from cedar forests in the Kanto plain of Japan
}

\author{
Q. Wang ${ }^{1}$, G. Otsuka ${ }^{1}$, S. Lu ${ }^{2}$, Y. Takai ${ }^{1}$, S. Dong ${ }^{1}$, M. Suzuki ${ }^{1}$, \\ D. Nakajima ${ }^{3}$, K. Sekiguchi ${ }^{1} \&$ M. Miwa ${ }^{4}$ \\ ${ }^{1}$ Graduate School of Science and Engineering, Saitama University, Japan \\ ${ }^{2}$ Shanghai University, China \\ ${ }^{3}$ National Institute for Environmental Studies, Japan \\ ${ }^{4}$ Center for Environmental Science in Saitama, Japan
}

\begin{abstract}
In recent years, pollinosis has been considered a "national disease" with the big social problems in urban areas of Japan. In this study, the airborne behavior of cedar pollen in the Kanto plain of Japan was observed by a real time pollen automatic measuring instrument during the 2014 pollination season. For calculating cedar pollen source contribution, the backward trajectory analysis was used for calculating 72 hours of trajectories of air mass with the altitude of $500 \mathrm{~m}$ from the urban of Saitama city to different areas. It was found that cedar pollen grains transported to Saitama city were mainly derived from the forest of Gunma prefecture located on the north side of Saitama city and the forest of Kanagawa prefecture located on the south side of the city. Transportation of cedar pollen grains depends on the weather conditions, habitat density of cedar trees and transportation distance. The variations in daily pollen counts among different transporting times showed three pollen dispersal peaks observed in the morning, the day time and the night time, while these occurrence rates were different in each month. The peak pollen counts in the day time correlated positively with the daily maximum temperature. The highest pollen counts were observed in the night time within three pollen dispersal peaks. At the same time, the size-segregated allergenic particles were also collected using Andersen high-volume air samplers at the roadside of urban residential zones of Saitama city and on the 10th floor of the research building in Saitama University. Allergenic
\end{abstract}


concentrations were measured by the surface plasmon resonance method with a Biacore J system. The highest airborne allergenic concentrations were observed in fine particles below $1.1 \mu \mathrm{m}$. Moreover, the forward trajectory analysis was used for calculating 72 hours of trajectories of air mass with the altitude of $500 \mathrm{~m}$ from different cedar pollen source areas including the rainfall events. It was suggested that not only cedar pollen grains but also its allergenic particles were transported from different cedar forests as the source areas.

Keywords: Japanese cedar pollinosis, Cryptomeria japonica pollen, allergenic particles, source contribution.

\section{Introduction}

In keeping the urban activity of the cities, a large amount of fossil energy and resources are consumed. As the result, discharge of greenhouse gases has been caused by fossil energy utilization. Nowadays, one of the reducing solutions for global warming - biomass resource - has attracted a lot of attention for its sustainability and carbon neutrality. In Japan, the cedar (Cryptomeria japonica) tree is the most useful woody biomass, and its forests have covered nearly $18 \%$ of the total of Japanese national forests and $44 \%$ of the total artificial forests [1]. However, artificial cedar forests are left decontroled and less utilized due to the decrease of silviculture workers for forest management. Moreover, 30 year old cedar trees which have the maximum production of cedar pollen remain unutilized. This problem causes Japanese cedar pollinosis which has had its highest prevalence rate $(26.5 \%)$ in Japan, from 2008 [2, 3]. Japanese cedar pollinosis not only causes a lowering in the quality of living level, but is also the main cause of "national disease" with the big social problems in urban areas of Japan. It has been reported that the declining labor productivity in urban residents in the Kanto plain of Japan had been caused by pollinosis [4]. In other words, sustainable city planning is related to forest management which is the balanced supply and demand of cedar trees for woody biomass in Japan. For utilizing cedar trees and countermeasure for its pollinosis, the main cedar pollen source area influencing an urban city should be identified. For those reasons, in this study, the airborne behavior of cedar pollen including the cedar pollen source contribution and its size-segregated allergenic concentrations in the urban area of Saitama city adjoining the north of Tokyo metropolitan area were investigated.

\section{Materials and methods}

\subsection{Airborne cedar pollen observation in Kanto plain of Japan in 2014}

In Japan, airborne pollen counts are typically measured by the Durham method. Airborne cedar pollen grains were collected gravimetrically by a glass coated fixed slide with petroleum jelly for 24 hours of sampling and counted using a microscope. The Durham method has been used for counting airborne pollen grains as a Japanese standard method up to now. However, it is considered that 
the method is not proper on airborne cedar pollen concentrations because it is only used for observation of pollen counts per square centimeter (counts $\left./ \mathrm{cm}^{2}\right)$. Therefore, the observation of airborne pollen counts per unit volume $\left(\right.$ counts $\left./ \mathrm{m}^{3}\right)$ is required. It accurately represents the degree of exposure effects on the human body respiratory system, such as Japanese environmental standards for suspended particulate matter (SPM) [5]. Recently, the real time pollen automatic measuring instruments (KH-3000-01, Yamatronics Co., Ltd., Japan) were installed by the Ministry of the Environment (MOE) of Japan used for the Japanese national forecast system for airborne pollen information known as "Hanakosan". Airborne cedar pollen information is provided in real time for public health and the reduction of pollinosis. This instrument introduces air (flow rate: $4.1 \mathrm{~L} / \mathrm{min}$ ) into a laser optical system which distinguishes pollen grains from other particles by forward and sideways-scattered light intensity and the number of pollen grains by the scattered light counter [6]. Real time pollen information is accessible on the MOE website and past observation data can be obtained [7]. The data of cedar pollen counts were observed at 7 sites such as Saitama city, Gunma prefecture, Tochigi prefecture, Ibaraki prefecture, Chiba prefecture, Kanagawa prefecture and Yamanashi prefecture located on the Kanto plain of Japan were included in this study which was carried out from February to April in 2014 (Figure 1).

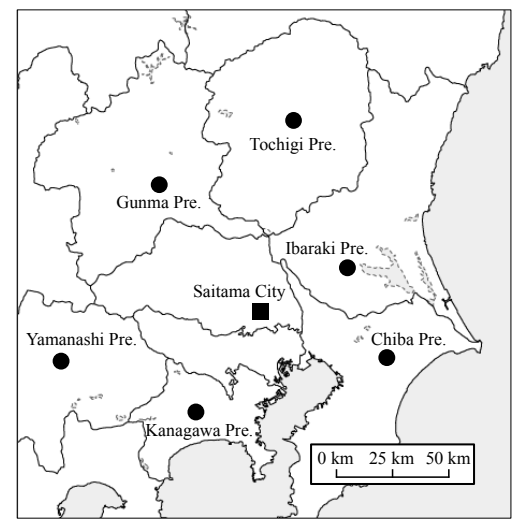

Figure 1: Airborne pollen observation sites located on the Kanto plain of Japan.

\subsection{Source contribution of cedar pollen grains}

In order to investigate the main cedar pollen source of Kanto plain, source contribution was calculated using hourly cedar pollen count data during the 2014 pollination season. In this study, pollen dispersal peaks were also observed. The number of pollen dispersal peaks and total pollen counts of dispersal peaks were used for calculating the pollen source contribution. As judgment with regard to pollen dispersal peaks, we defined the pollen peaks as pollen counts more than 50 counts $/ \mathrm{m}^{3}$ from February to the beginning of March or more than 200 
counts $/ \mathrm{m}^{3}$ after the middle of March. This definition was based on the pollen dispersal trend in this research period. In the case of peak pollen counts of less than 50 counts $/ \mathrm{m}^{3}$ or 200 counts $/ \mathrm{m}^{3}$, it was also considered as a pollen dispersal peak because of the obvious increase in pollen counts before and after counting.

In the trajectory analysis, backward trajectories were used to show the path taken by air mass containing cedar pollen as they approached Saitama city. The backward trajectory analysis of 72 hours of trajectories of air mass with the altitude of $500 \mathrm{~m}$ was calculated from the urban area of Saitama city to different areas during pollen dispersal peaks observation. In contrast, forward trajectories were used to show where air mass containing cedar pollen travelled from the each cedar forest on the Kanto plain. The forward trajectory analysis was used for calculating 72 hours of trajectories of air mass with the altitude of $500 \mathrm{~m}$ from different cedar pollen source areas including the rainfall events. We calculated trajectories with the computer software of CGER-METEX (Meteorological Data Explorer) which was developed by the Center for Global Environmental Research (CGER) of the National Institute for Environmental Studies (NIES), Japan. METEX software can be downloaded from the website of NIES-CGER [8].

\subsection{Meteorological data}

Meteorological data was downloaded from Automated Meteorological Data Acquisition System (AMeDAS) observed by the Japan Meteorological Agency at 7 sites during the 2014 study period [9]. Temperature [ $\left.{ }^{\circ} \mathrm{C}\right]$, rainfall [mm], wind direction and wind speed $[\mathrm{m} / \mathrm{s}]$ were considered as the measurement items in the system.

\subsection{Measurement of airborne pollen and allergenic particles in Saitama city}

In order to determine the airborne cedar pollen and its size-segregated allergenic concentrations in Saitama city, the roadside sampling site was selected near Japan national route 463 in the urban residential zone of Saitama city where the traffic density is around 25,000 vehicles per day [10]. At the same time, the general urban sampling site on 10th floor of the research building of Saitama University was also decided. From the roadside sampling, it may be possible to investigate both the influences of the exhaust gas emitted by automobiles and the re-scattering pollen grains and their allergenic particles by vehicle transportation. However, it is considered that the general urban sampling site on the 10th floor of the building is less influenced by automobile traffic. Daily airborne cedar pollen was collected by the Durham sampler (DK-1SA, Nishiseiki Co. Ltd., Japan) instead of KH-3000-01 in order to count deformation or denature cedar pollen grains. After 24 hours of sampling, for counting the cedar pollen grains, we firstly prepared Phöbus Blackly stain solution [11], dropped it onto the glass slide samples and then the pollen grains were counted using a microscope and calculated pollen counts per square centimeter (pollen counts $/ \mathrm{cm}^{2}$ ). The sampling period was scheduled to be from 26th March to 24th April in 2014. At the same time, airborne allergenic particles were collected on the quartz fiber filters by an 
Andersen high-volume air sampler (AH-600, Shibata Scientific Technology Co. Ltd., Japan). This instrument collected the atmospheric suspended particulate matter equivalent to the human respiratory system as five size-segregated stages such as alveo: below $1.1 \mu \mathrm{m}$, bronchial branch: 1.1-2.0 $\mu \mathrm{m}$, bronchium: 2.0-3.3 $\mu \mathrm{m}$, pharynges and throat: $3.3-7.0 \mu \mathrm{m}$, mouth and nasal cavity: above $7.0 \mu \mathrm{m}$. The air flow of the sampler was $566 \mathrm{~L} / \mathrm{min}$. The filters were changed every 47 hours (26th-27th March and 28th-29th March) or every 167 hours (30th March-4th April, 5th-10th April, 11th-17th April, and 18th-24th April). Allergenic concentrations were measured by the surface plasmon resonance method with a Biacore J system described previously [11-13]. The filters collected from airborne particles were cut out and put into centrifuge tubes (polypropylene copolymer), followed by the addition of $2 \mathrm{~mL}$ of pollen extracting solution $[12,13]$. After incubation of 24 hours at $4{ }^{\circ} \mathrm{C}$, the centrifuge tubes were followed by centrifugation at $6,000 \mathrm{rpm}$ for 30 minutes at room temperature using the centrifuge ( $\mathrm{CN}-1050$, AS ONE Corp., Japan). The supernatants were collected and analyzed. Allergenic concentrations were measured by using the Cry $\mathrm{j} 1$ polyclonal antibody (Anti-Cry $\mathrm{j} 1$ rabbit IgG fraction, Hayashibara Co. Ltd., Japan) which was immobilized on a CM5 sensor chip (GE Healthcare Co. Ltd., Japan). Approximately $400 \mathrm{ng}$ of antibody was immobilized on the chip. The regeneration and dissociation of the antigen-antibody complex reaction on the CM5 sensor chip were performed by $70 \mu \mathrm{L}$ of $\mathrm{pH} 2.0$ glycine-HCl (GE Healthcare Co. Ltd., Japan). Finally, the results were converted into allergenic concentrations $\left(\mathrm{ng} / \mathrm{m}^{3}\right)$ in the urban atmosphere.

\section{Results and discussions}

\subsection{Trend of airborne cedar pollen dispersal in Saitama city in 2014}

Daily average pollen counts in Saitama city during the 2014 pollination season are shown in Figure 2. Generally, it is considered that cedar pollen will start scattering on the Kanto plain when the integrated total value of daily maximum temperature from 1st January reaches $300^{\circ} \mathrm{C}$ [14]. During the 2014 study period, the integrated value of the daily maximum temperature reached $300^{\circ} \mathrm{C}$ at the beginning of February at each observation site. After this point, daily pollen counts increased toward the pollen dispersal peak period which was after mid-March. Fortunately, the pollen dispersal peak period was the same time as our airborne particle sampling in this study.

The monthly variation of average cedar pollen counts in Saitama city and Gunma prefecture in recent 5 years is shown in Figure 3. It was considered that monthly airborne cedar pollen counts in 2014 were as well as in recent 5 years. The highest cedar pollen counts were observed in 2013 in the Gunma prefecture. The production of cedar pollen was related to temperature and amount of insolation in the previous summer. High temperature and long hours of sunlight will promote flower initiation which results in more cedar pollen dispersal [14, 15]. In 2012, the summer temperature and amount of insolation were $+0.7^{\circ} \mathrm{C}$, $110 \%$ in July, $+1.4^{\circ} \mathrm{C}, 137 \%$ in August and $+2.0^{\circ} \mathrm{C}, 129 \%$ in September, 
respectively, on the Kanto plain compared with the average values based on 30 years' data. The monthly variation of average cedar pollen counts in 2013 were influenced by these meteorological conditions. However, decreasing pollen counts in 2014 were considered as a rebound reaction of last large scattering year. This trend was shown prominently in Gunma prefecture near the mountain area located on the north side of Saitama city.

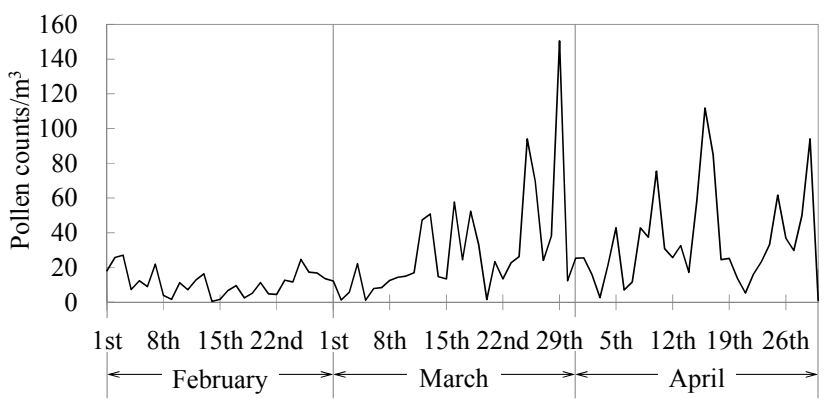

Figure 2: Daily average pollen counts in Saitama city during the 2014 pollination season.
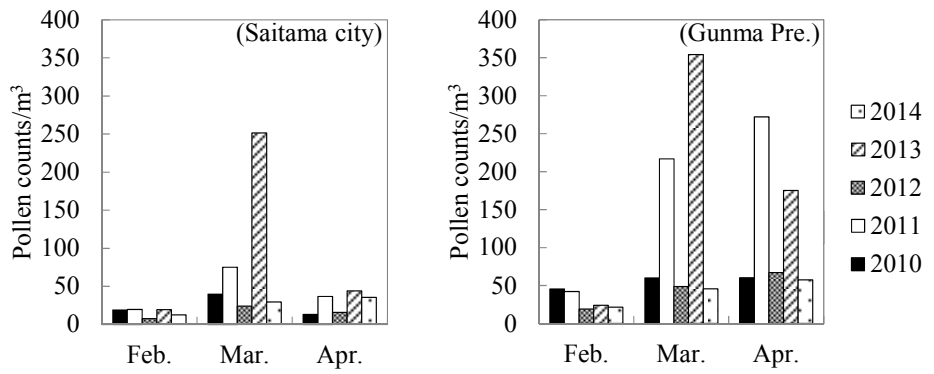

Figure 3: Monthly variation of average cedar pollen counts in Saitama city and Gunma prefecture in the recent 5 years.

Pollen dispersal peaks in hourly pollen counts could be classified as three time periods in the morning (5:00-10:00JST), the day time (10:00-18:00JST) and the night time (18:00-following 5:00JST). Monthly total peak pollen counts of three pollen dispersal types in Saitama city are shown in Figure 4. Airborne pollen counts are affected by weather conditions such as temperature, wind, relative humidity and rainfall. It was reported that high temperature and low relative humidity promote more cedar pollen dispersal during the day time $[16,17]$. In this study period, peak pollen counts in the day time were correlated positively $(\mathrm{r}=0.73, \mathrm{p}<0.0005)$ with the daily maximum temperature. The pollen counts in the night time in March were the highest among dispersal peaks. Within the dispersal peaks in the morning, March was the highest pollen counts period over three months. Due to the absence of cedar pollen source in Saitama city, cedar pollen grains were transported from a mountain area on Kanto plain. Furthermore, the dispersal peaks in the morning and the night time are caused by 
the atmospheric stabilization in the evening, since atmospheric stabilization is caused by a decreasing amount of insolation and temperature. With rising temperature during the day time, cedar pollen grains were released more and taken high up into the atmosphere through convection [18, 19]. Suspended cedar pollen grains descend slowly towards ground level and are concentrated due to weak turbulence [17]. It was reported that dispersal peaks in the night time in the urban area of Kanto Plain were produced by transportation of pollen grains which were released from the mountain areas during the day time [20]. In this study period, the dispersal peaks in the night time and in the morning which were observed in Saitama city delayed 4 and 8 hours after pollen grains were released from the cedar forests as its pollen sources. Moreover, re-scattering pollen grains from the ground were promoted by increasing vehicle transportation and turbulence of convection in the morning [21].

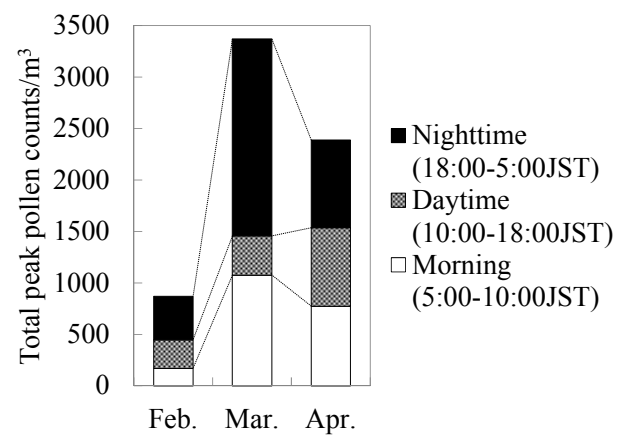

Figure 4: Monthly total peak pollen counts of three pollen dispersal types in the morning, the day time and the night time in Saitama city during 2014.

\subsection{Cedar pollen source contribution from each forest of Kanto plain}

Due to the absence of a cedar pollen source in Saitama city, cedar pollen grains were transported from the mountain areas on Kanto plain. The backward trajectory analysis was used for calculating 72 hours of trajectories of air mass with the altitude of $500 \mathrm{~m}$ from the urban area of Saitama city to different areas when pollen dispersal peaks were observed. From the results of backward trajectories shown in Figure 5, it was found that the cedar forests as its pollen sources can be contributed from 5 sampling sites such as Gunma, Kanagawa, Tochigi, Ibaraki, and Yamanashi prefectures. However, a cedar pollen source contribution of Chiba prefecture was not found in the trajectories analytical results. Pollen source contribution on the number of pollen peaks and total peak pollen counts from different sites during the 2014 sampling period from February to April are shown in Figure 6.

It was found that the northwest wind occurred mainly during the cedar pollen scattering season [22]. This prevailing wind direction resulted in the highest contribution of the forest of the Gunma prefecture among cedar pollen sources. The second highest contribution was recorded in the forest of Kanagawa prefecture. Therefore, the forest of the Gunma and Kanagawa prefectures were 
considered as the main cedar pollen source. It means that airborne cedar pollen observed in Saitama city was mainly transported from the forest of Gunma and Kanagawa prefectures. In contribution on number of peaks, the contribution of the forest of Yamanashi prefecture was lower than that of Tochigi prefecture. In contrast, contribution on total peak pollen counts of Yamanashi prefecture was higher than that of Tochigi prefecture. The forest densities of Kanagawa and Yamanashi prefectures are higher than those of Tochigi and Ibaraki prefectures [19]. These results were affected by the habitat density of cedar trees. Since Chiba prefecture is located in the southeast of Kanto plain besides the sea, the pollen contribution of the forest of Chiba prefecture was not observed during this study period.
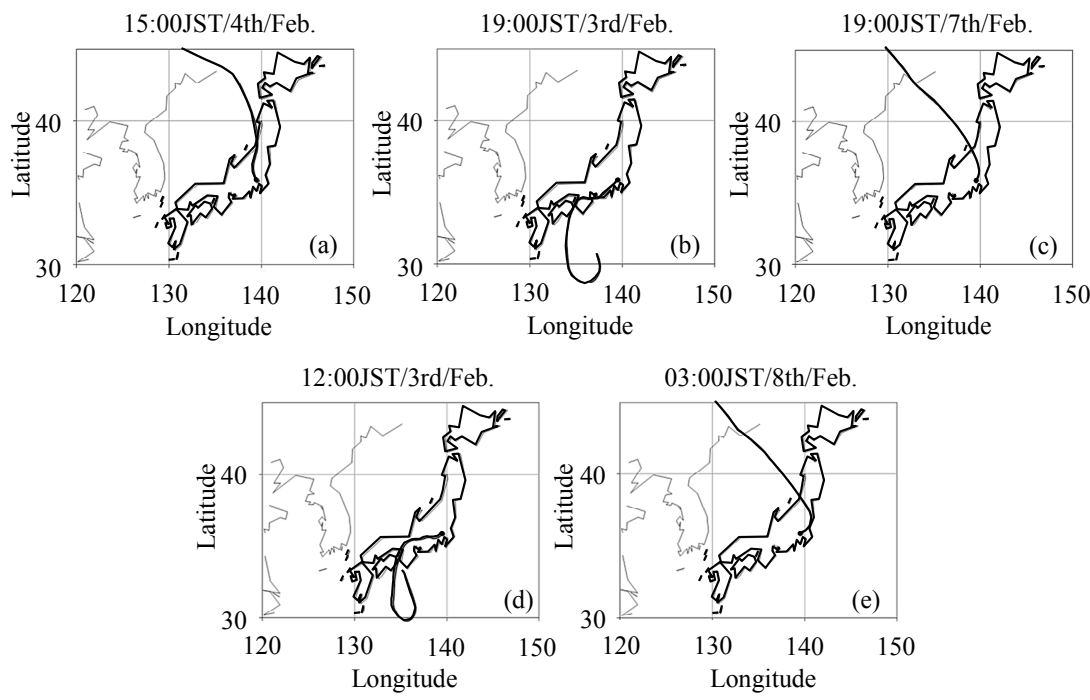

Figure 5: The results of 72 hours of backward trajectories drawn by METEX (an altitude of $500 \mathrm{~m}$ ) from the urban of Saitama city to 5 sampling sites: (a) Gunma prefecture, (b) Kanagawa prefecture, (c) Tochigi prefecture, (d) Yamanashi prefecture, (e) Ibaraki prefecture in the 2014 sampling period.

The monthly contribution on total peak pollen counts can be classified as three pollen dispersal types (shown in Figure 7). And the monthly variation of total peak pollen counts observed in Saitama city or in pollen source areas are shown in Figure 8. The high pollen contribution of the forest of Gunma and Kanagawa prefectures was given in Figure 6. The pollen source contribution of the forest of these prefectures was recorded in almost peak times. Though the total peak pollen counts observed in Saitama city correlated with the pollen source areas, the pollen counts were about one half of pollen source areas. This difference in the pollen counts between Saitama city and pollen source areas could be influenced by the diffusion and decreasing airborne cedar pollen. 

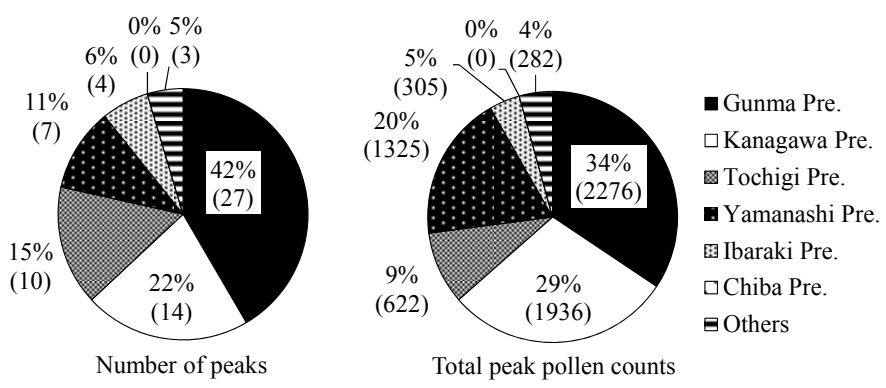

Figure 6: Contribution on number of peaks and on total peak pollen counts from February to April in the 2014 sampling period.

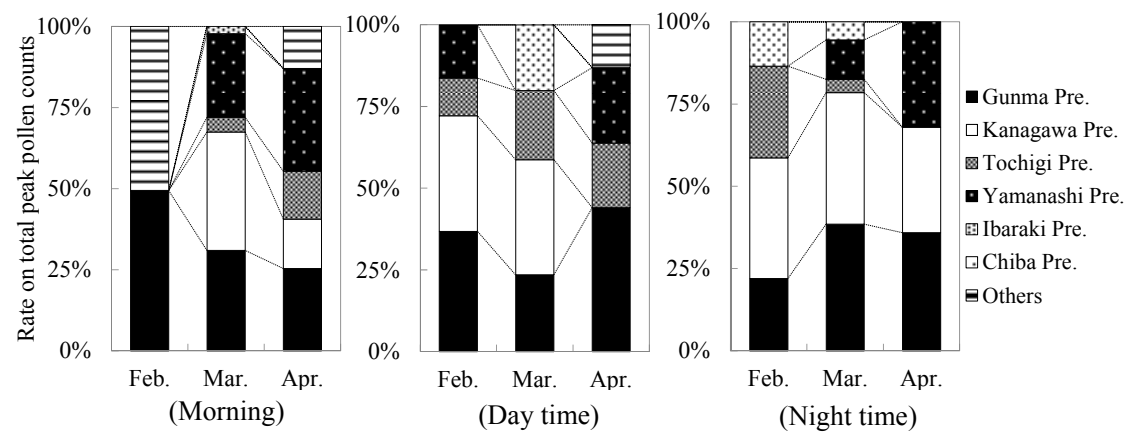

Figure 7: Monthly contribution on total peak pollen counts in the three classified pollen dispersal types in the 2014 study period.

It was observed that airborne pollen grains were deposited in the night time and the re-scattering of pollen grains were promoted by increasing vehicle transportation and turbulence of convection in the morning. In the day time, pollen source contribution of the forest of Kanagawa prefecture was higher than that of Gunma prefecture during February to March. It was previously reported that airborne pollen counts were lower with northern wind than that with southern wind because of the diffusion and decreasing airborne cedar pollen grains with the distance of transportation from pollen source areas [20].

For the effective utilization of cedar trees and decrement of pollen dispersal around the urban city, it is important to take a countermeasure against main cedar pollen sources such as Gunma prefecture and Kanagawa prefecture.

\subsection{Size-segregated allergenic concentrations in Saitama city}

Cedar pollinosis is mainly caused by its allergen particles such as Cry $\mathrm{j} 1$. Airborne size-segregated allergenic concentrations in Saitama city are shown in Figure 9. Allergenic concentrations observed in coarse particles above $7.0 \mu \mathrm{m}$ are considered derived from cedar pollen grains, and particle sizes below $7.0 \mu \mathrm{m}$ is considered as micro allergenic particles which are released from cedar pollen [23]. During 26th-27th March, the highest allergenic concentrations 


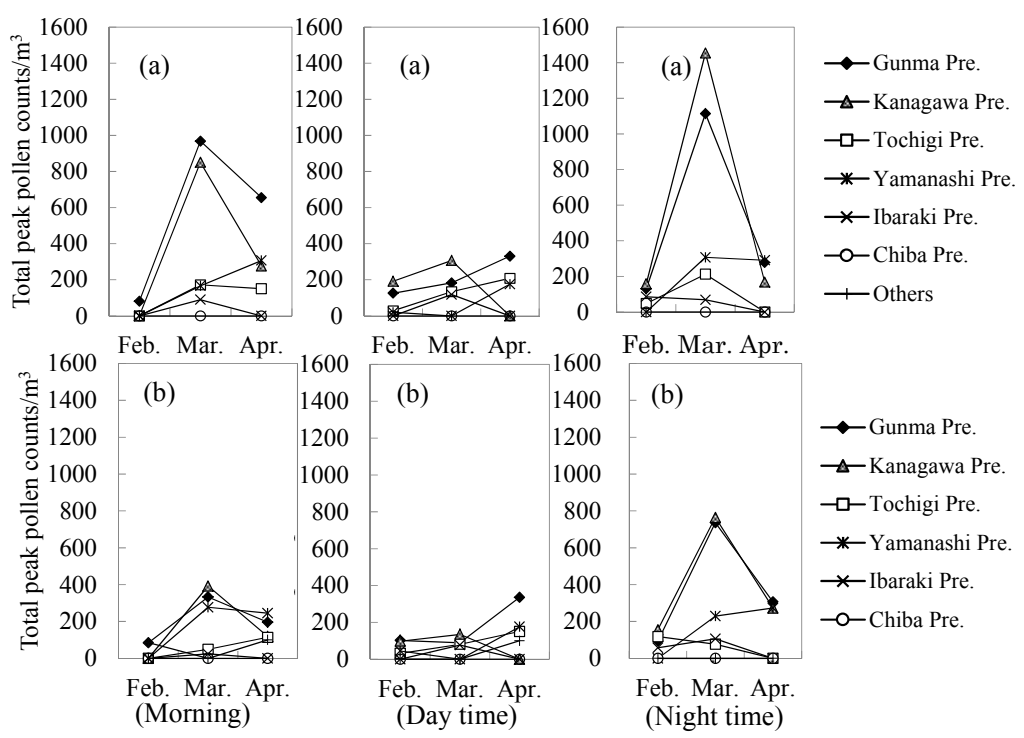

Figure 8: Monthly variation of total peak pollen counts in the three classified pollen dispersal types in pollen source areas (a) and in Saitama city (b) in the 2014 study period.

$\left(12 \mathrm{ng} / \mathrm{m}^{3}\right)$ observed in fine particles below $1.1 \mu \mathrm{m}$ at the roadside during the sampling period, with which it is possible to induce allergenic asthma. The micro allergenic particles released from cedar pollen grains were produced by rainfall and especially exposed to polluted air in this case. Cedar pollen allergen such as Cry $\mathrm{j} 1$ exposed to urban polluted air can develop a very strong bimolecular interaction with the anti-Cry j 1 antibody [24]. It means that the highest allergenic concentrations in this period were caused by urban polluted air. The symptom of pollinosis appeared when cedar pollen allergenic concentrations reach about $1-3 \mathrm{pg} / \mathrm{m}^{3}$ in the atmosphere [12]. Therefore, worsening asthmatic and pollinosis symptoms may occur in this period. Moreover, it was possible to transport allergenic particles from a pollen source area. The forward trajectory analysis was used for calculating 72 hours of trajectories of air mass with the altitude of $500 \mathrm{~m}$ from different cedar pollen source areas including the rainfall events. On 27th March, air masses were transported from Yamanashi and Kanagawa prefectures while a rainfall event was also observed.

In the latter half of the sampling period, although airborne pollen counts were at the same levels between the roadside and the 10th floor, allergenic concentrations at the roadside were lower than those on the 10th floor. It is considered that allergenic particles were derived from the outside area of Saitama city. Therefore, a forward trajectory analysis was also carried out. As a result, air masses containing pollen allergenic particles transported from different pollen sources after rainfall events were not only observed in Yamanashi and Kanagawa prefectures during 5th-10th April, but were also observed in all observation sites on Kanto Plain during 18th-24th April, 2014. 


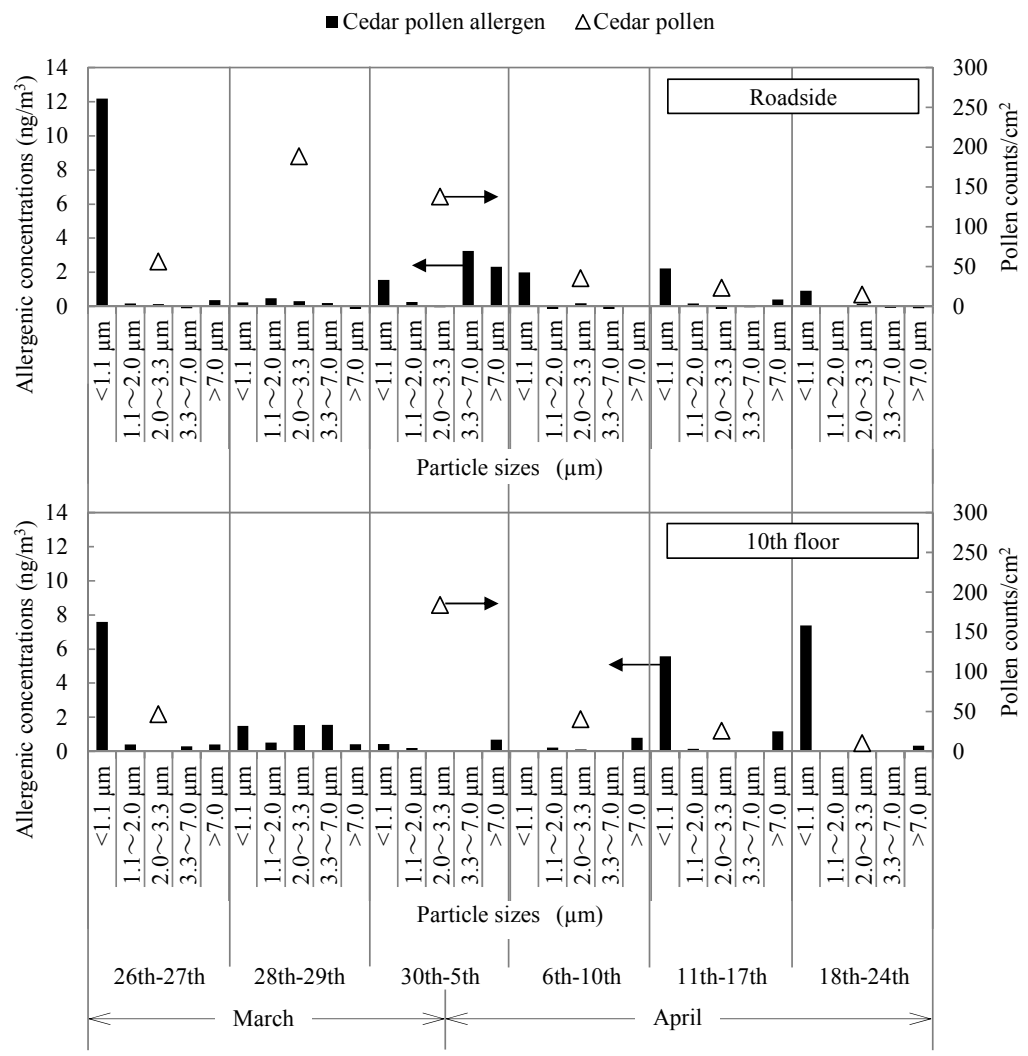

Figure 9: Allergenic concentrations and cedar pollen counts at the roadside of Route 463 and on the 10th floor of the research building in the 2014 sampling period.

\section{Conclusion}

In this study, we reported the airborne behavior of cedar pollen and its allergen released from the cedar forest of different prefectures located on the Kanto plain of Japan. Cedar pollen dispersal will be affected by weather conditions, habitat density of cedar trees and the distances of transportation. Cedar pollen grains transported to Saitama city were mainly derived from the Gunma prefecture located on the north side of Saitama city and Kanagawa prefecture located on the south side of the city. From the observation of hourly pollen counts, it was found that there are three types of pollen dispersal peaks in the morning, the day time and the night time. The peak pollen counts in the day time correlated positively with the daily maximum temperature. The peak pollen counts in the night time were the highest in three types of pollen dispersal peaks. It means that airborne pollen grains were transported with convection in the day time and concentrated 
at ground level due to weak turbulence and atmospheric stabilization in the night time. The highest airborne allergenic concentrations were determined in fine particles below $1.1 \mu \mathrm{m}$. It is suggested that these fine allergenic particles may easily cause allergenic asthma in the urban area of Japan. Moreover, with the help of trajectory analysis, it was suggested that not only cedar pollen grains but also allergenic particles were transported from the main pollen source area. For reducing pollinosis symptoms in the urban area of Japan, it is necessary to consider suitable city planning with the help of the effective utilization of cedar trees and decrement of pollen dispersal around the urban areas.

\section{Acknowledgements}

Some works of this study are supported by the Special Funds for Grant-in-Aid for Scientific Research (B) (No. 24310005, FY 2012-FY2014) and of the Japanese Ministry of Education, Culture, Sports, Science and Technology (MEXT).

\section{References}

[1] Forestry Agency, Forestry White Paper (2013) (in Japanese).

[2] Murayama, K. et al., Regional differences in the prevalence of cedar-pollen allergy. Japanese Society of Allergology 59, 47-54 (2010) (in Japanese).

[3] Kitamura T. et al., Allergy (1): Nasal allergy by pollen, Earozoru Kenkyu, 20(1), 54-57 (2005).

[4] Minami, Y. et al., Japanese Cedar pollinosis impact on work productivity, quality of life and symptoms 2008 vs. 2009, Nihon Bika Gakkai Kaishi, 49(4), 481-489 (2010).

[5] The Ministry of the Environment, Environmental standards for air pollution (last access: May 5, 2015), http://www.env.go.jp/kijun/taiki.html (in Japanese)

[6] Yoda S. et al., A comparative study between real time monitor KH-3000 and conventional Durham sampler measuring airborne pollen, Nihon Jibiinkoka Gakkai Kaiho, 108(8), 801-805 (2005).

[7] The Ministry of the Environment, pollen observation system (called Hanakosan) (last access: May 5, 2015), http://kafun.taiki.go.jp/ (in Japanese).

[8] National Institute for Environmental Studies-Center for Global Environmental Research, CGER-METEX http://db.cger.nies.go.jp/metex/ trajectory.jp.html (in Japanese) (last access: May 5, 2015).

[9] Japan Meteorological Agency, AMeDAS, (last access: May 5, 2015), http://www.jma.go.jp/jma/index.html (in Japanese).

[10] Saitama Prefecture, Japan, National road and street traffic situation investigation (2005) (in Japanese).

[11] Wang, Q. et al., Release behavior of small sized daughter allergens from Cryptomeria japonica pollen grains during urban rainfall event, Aerobiologia, 28(1), 71-81 (2012). 
[12] Takahashi, Y. et al., Possibility of real-time measurement of an airborne Cryptomeria japonica pollen allergen based on the principle of surface plasmon resonance, Aerobiologia, 17, 313-318 (2001).

[13] Araki, T. et al., Analysis of pollen dispersion in Chugoku-Shikoku region due to the information from the monitoring network system of the Ministry of the Environment, Journal of Environmental Laboratories Association, 35(1), 25-32 (2010) (in Japanese).

[14] Takahashi, Y. et al., Identification of airborne pollen and airborne particles with pollen allergen (Cry j 1, Dac g) by aeroallergen immunoblotting technique, Japanese Journal of Allergology, 51, 609-614 (2002).

[15] Sahashi, N. et al., Review of the pollen front of Cryptomeria japonica over japan, Jpn. J. Palynol, 41(2), 119-1217 (1995).

[16] Bartkova-Scevkova, J., The Influence of Temperature, Relative Humidity and Rainfall on the Occurrence of Pollen Allergens (Betula, Poaceae, Ambrosia artemisiifolia) in the atmosphere of Bratislava (Slovakia), International Journal of Biometeorology, 48, 1-5 (2003).

[17] Claudio F et al., Comparison of intradiurnal variation of airborne pollen in Mar del Plata (Argentina). Part I. Non-arboreal pollen. Aerobiologia, 17, 151-163 (2001).

[18] B. Sikoparija et al., A mechanism for long distance transport of ambrosia pollen from the Pannonian plain. Agricultural and Forest Meteorology, 180, 112-117 (2013).

[19] Kanazashi, T. \& Suzuki, M., The estimation of the pollen source areas of Cryptomeria japonica D. Don which have high contributions to the Japanese metropolitan region, The Japanese Forest Society, 92, 298-303 (2010) (in Japanese).

[20] Wang, Q. et al., Diurnal and nocturnal behaviour of airborne cryptomeria japonica pollen grains and the allergenic species in urban atmosphere of Saitama, Japan, Asian Journal of Atmospheric Environment, 7(2), 65-71 (2013).

[21] Yoshii, E. et al., Observations of airborne Cryptomeria japonica pollen in the summer, Allergology International, 50, 171-177 (2001).

[22] Takahashi, H., Climatological feature of diurnal variation in wind systems and divergence field over Kanto district under stable synoptic conditions -in the cases of summertime land-sea breezes and strong winter monsoon-, The New Geography, 46(1), 104b-104b (1998).

[23] Wang, Q. et al., Daily Variation Differences between Airborne Japanese Cedar Pollen and its Respirable Allergen Particulate Matters during the Most Severe Pollination Seasons. Earozoru Kenkyu 23, 120-126 (2008) (in Japanese).

[24] Wang, Q. et al., Characterization of the physical form of allergenic Cry $\mathrm{j} 1$ in the urban atmosphere and determination of Cry $\mathrm{j} 1$ denaturation by air pollutants, Asian Journal of Atmospheric Environment, 6(1), 33-40 (2012). 